\title{
EL "AULA INVERTIDA" COMO METODOLOGÍA ACTIVA PARA FOMENTAR LA CENTRALIDAD EN EL ESTUDIANTE COMO PROTAGONISTA DE SU APRENDIZAJE
}

\author{
Francisco Javier Domínguez Rodríguez \\ Ascensión Palomares Ruiz \\ Universidad de Castilla La Mancha
}

\begin{abstract}
RESUMEN: Los procesos de enseñanza-aprendizaje en los títulos universitarios y, especialmente, en los títulos de Grado, están evolucionando hacia una mejora de los contenidos digitales y el uso de plataformas de aprendizaje y gestión académica. En este sentido, las "aulas invertidas" o "flipped classroom" consisten en darles la vuelta a las clases tradicionales con el fin de convertir al alumnado en protagonista activo de su aprendizaje. En la asignatura del segundo curso del Grado de Maestro/a de Educación Primaria denominada Tendencias Contemporáneas de la Educación se ha implementado esta metodología. El objetivo de este trabajo consistió en realizar un pilotaje en dicha asignatura y evaluar el grado de satisfacción del alumnado sobre dicha metodología. Para ello, se utilizó el cuestionario de percepciones de los estudiantes sobre la clase invertida (Gilboy et al., 2015). Los resultados obtenidos demuestran que los estudiantes prefieren participar en clase a través de la realización de trabajos activos y grupales que una clase tradicional. La aplicación de este modelo didáctico favorece un mayor aprovechamiento del tiempo en el aula, el uso de las Tecnologías de la Información y Comunicación y la colaboración entre estudiantes, la motivación y la centralidad en el alumnado como protagonista de su propio aprendizaje.
\end{abstract}

PALABRAS CLAVE: Educación superior, aula invertida, enseñanzaaprendizaje, percepción estudiantes.

\section{THE "FLIPPED CLASSROOM" AS AN ACTIVE METHODOLOGY TO PROMOTE CENTRALITY IN THE STUDENTS AS PROTAGONIST OF THEIR OWN LEARNING}

\footnotetext{
ABSTRACT: Teaching-learning processes in university degrees, and especially graduate degrees, are evolving towards a digital contents
} 
improvement and the use of learning platforms and academic management. In this sense, the "flipped classroom" lies in turning the traditional lessons in order to make students the active protagonists of their own learning. In the subject called Contemporary Trends in Education studied on the second year of the Primary Education Teacher Degree, this methodology has been implemented. The objective of this work was to experience this subject and assess the degree of student satisfaction in this methodology. In order to do this, the questionnaire about student perceptions regarding the flipped classroom was used (Gilboy et al., 2015). The results demonstrate that students prefer to participate in class through active and group work rather than in a traditional class. The application of this educational model promotes a better exploitation of time in the classroom, the use of Information and Communication Technologies and collaboration between students, motivation and focus on students as the protagonists of their own learning.

KEYWORDS: Higher education, flipped classroom, teaching-learning, perception students.

Recibido: $12 / 06 / 2020$

Aceptado: 03/07/2020

Correspondencia: Francisco Javier Domínguez Rodríguez. Universidad de Castilla La Mancha. Facultad de Educación de Ciudad Real. Ronda de Calatrava, 3, 13071, Ciudad Real. Email: Francisco.Dominguez@uclm.es

\section{INTRODUCCIÓN}

El proceso de convergencia europea ha supuesto un cambio radical en el paradigma del proceso enseñanza-aprendizaje universitario, por lo que el Espacio Europeo de Educación Superior (en adelante EEES) Ilevó aparejado la transformación en los roles educativos, pasando de una docencia basada en la clase magistral a una docencia centrada en el alumnado (Reyes, 2015). Tal y como señalan Santiago y Bergmann (2018), "si analizamos estas reformas descubriremos que los aspectos en los que se han centrado son, por un lado, el currículo-los contenidos, lo que se enseña-y, por otro, la evaluación-cómo comprobar lo que los alumnos saben" (p. 7).

Actualmente, uno de los aspectos principales en los procesos de enseñanzaaprendizaje es poder favorecer un aprendizaje activo y lograr ofrecer un mayor protagonismo al alumnado, todo ello acompañado del uso de las Tecnologías de la Información y Comunicación. En este sentido, Bergmann y Sams (2014) sostienen que es primordial que el estudiante tome responsabilidad de su propio aprendizaje, ya que cuando el docente otorga el control del proceso de aprendizaje, este alumnado toma el mando y el proceso educativo se desarrolla más fácilmente. Asimismo, Bergmann 
y Sams (2012) indican que, en el proceso de enseñanza-aprendizaje, el alumnado precisa de la presencia física del docente, en el momento preciso que se atascan en un contenido y necesitan su ayuda.

Razonadamente, la metodología del "flipped classroom" o "aula invertida" está teniendo un gran protagonismo, debido a que aúna las principales tendencias educativas: el aprendizaje activo y uso de las Tecnologías de la Información y Comunicación (Hernández y Tecpan, 2017). Igualmente, cada día, cobra mayor importancia, en la sociedad del conocimiento y de la información, el avance progresivo de la tecnología, la cual ha incidido inevitablemente en el ámbito educativo, facilitando el acceso a la información al alumnado. Este avance ha logrado que el estudiante pueda autorregular su propio aprendizaje a partir del uso de dispositivos móviles (Aznar et al., 2018). Consecuentemente, diversos autores han insistido en la aplicación de este modelo didáctico o metodología activa en el contexto de la enseñanza superior (Tucker, 2012; Ash, 2012; Cebrián et al., 2019).

En esta línea, el presente estudio tiene el objetivo general de realizar un pilotaje en la asignatura Tendencias Contemporáneas de la Educación del Grado de Maestro de Educación Primaria de la Universidad de Castilla-La Mancha para evaluar el grado de satisfacción del alumnado sobre la metodología "flipped classroom" y, a su vez, otros específicos como ver si mejora su rendimiento académico, destacar recursos que facilitan el proceso de aprendizaje, así como analizar las ventajas y los inconvenientes que percibe con la implementación de esta metodología.

\section{Concepto y desarrollo del "aula invertida" o "flipped classroom"}

Resulta importante subrayar algunas de las conceptualizaciones de "flipped classroom" de los autores que más han desarrollado esta metodología. Así, destacamos a Bergmann y Sams (2014), que al observar que los estudiantes, en determinadas situaciones, perdían clases por diferentes razones y para ayudar a dicho alumnado, impulsaron la grabación y distribución de videos. Además, se dieron cuenta que este modelo permitía al profesorado centrarse más en la atención a las necesidades individuales de aprendizaje de cada estudiante. Por ello, acuñaron el término "flipped classroom" y lo conceptualizaron como un enfoque pedagógico, en el que la instrucción directa se desplaza de la dimensión del aprendizaje grupal a la dimensión del aprendizaje individual, transformándose el espacio grupal restante en un ambiente de aprendizaje dinámico e interactivo en el que el facilitador guía al alumnado en la aplicación de los conceptos y en su implicación creativa con el contenido del curso. Visiblemente, el denominado "flipped classroom", es una de las metodologías didácticas que están emergiendo en este último lustro, que surgen alrededor del uso de las nuevas tecnologías e intentan aprovechar todo su potencial comunicativo, informacional, colaborativo, interactivo, creativo e innovador en el marco de una nueva cultura del aprendizaje. "El proceso de aprendizaje ya no se reduce a una mera transmisión de datos, sino a una misión puramente creativa" (Santiago y Bergmann, 2018, p. 12).

Coincidimos con las premisas de Tourón (2013), al señalar que nos encontramos con un modelo didáctico en el que el alumnado aprende a través de video-tutoriales 
en línea, habitualmente en casa; y lo que antes solían ser los "deberes" (tareas asignadas), se realizan ahora en el aula con el docente, quien facilita una orientación más personalizada y posibilita mayor interacción con los estudiantes. En esta misma línea, Santiago (2013) subraya que esta metodología transfiere el trabajo de determinados procesos de aprendizaje fuera del aula y utiliza el tiempo de clase, junto con la experiencia del docente, para facilitar y potenciar en el estudiante otros procesos de adquisición y práctica de conocimientos.

Razonadamente, mientras que en la enseñanza tradicional destaca la clase magistral en la que el mayor tiempo de permanencia se da en el aula y en la que se dedica a exponer y explicar la materia al alumnado para, posteriormente, indicar las tareas a realizar en casa; el modelo didáctico del "flipped classroom" transforma la metodología de enseñar, ya que es en casa donde el alumnado accede a los contenidos y las tareas se desarrollan dentro del aula (Tabla 1).

Tabla 1. Comparación entre aula tradicional y aula inversa (flipped classroom)

\begin{tabular}{|l|l|l|l|}
\hline \multicolumn{2}{|c|}{ AULA TRADICIONAL } & \multicolumn{2}{c|}{ AULA INVERSA } \\
\hline \multicolumn{1}{|c|}{ Actividad } & \multicolumn{1}{c|}{ Tiempo } & \multicolumn{1}{c|}{ Actividad } & \multicolumn{1}{c|}{ Tiempo } \\
\hline Actividad de calentamiento & $5 \mathrm{~min}$ & Actividad de calentamiento & $5 \mathrm{~min}$ \\
\hline $\begin{array}{l}\text { Repaso anterior (Tareas de } \\
\text { casa) }\end{array}$ & $20 \mathrm{~min}$ & $\begin{array}{l}\text { Preguntas y respuestas so- } \\
\text { bre el video }\end{array}$ & $10 \mathrm{~min}$ \\
\hline $\begin{array}{l}\text { Lectura y trabajo de nuevo } \\
\text { contenido }\end{array}$ & $30-45 \mathrm{~min}$ & $\begin{array}{l}\text { Actividad práctica y/o labo- } \\
\text { ratorio guiado e indepen- } \\
\text { diente }\end{array}$ & $75 \mathrm{~min}$ \\
\hline $\begin{array}{l}\text { Actividad práctica y/o labo- } \\
\text { ratorio guiado e indepen- } \\
\text { diente }\end{array}$ & $20-35 \mathrm{~min}$ & \\
\hline
\end{tabular}

Fuente: Traducido de Bergmann y Sams (2012).

En el modelo didáctico del "flipped classroom", el docente facilita a su alumnado una serie de materiales y recursos audiovisuales de corta duración (entre cinco y diez minutos) en los cuales se recogen los conceptos principales que el docente quiere que el alumnado trabaje y aprenda en cada una de los temas o unidades didácticas que se trabajan en la asignatura. Dicho material proviene de la red y también es material elaborado por el profesorado de la asignatura para completar conceptos que no se describen en los materiales en red. Por todo ello, los docentes universitarios que han adoptado e investigado sobre el modelo de aula invertida coinciden en señalar entre sus ventajas la de producir una mayor implicación del estudiante, un aprendizaje más profundo y permite mayor adaptación al ritmo de cada estudiante. En este sentido, Palomares y Cebrián (2016), destacan que el "flipped classroom" o "aula invertida" puede ser la palanca para el cambio, tanto en la universidad, como en el concepto del paradigma de la enseñanza global, construyendo una formación más holística y de calidad. Igualmente, el modelo "flipped classroom" permite invertir un mayor tiempo en clase en las categorías superiores de la taxonomía de Bloom (Figura 1). 


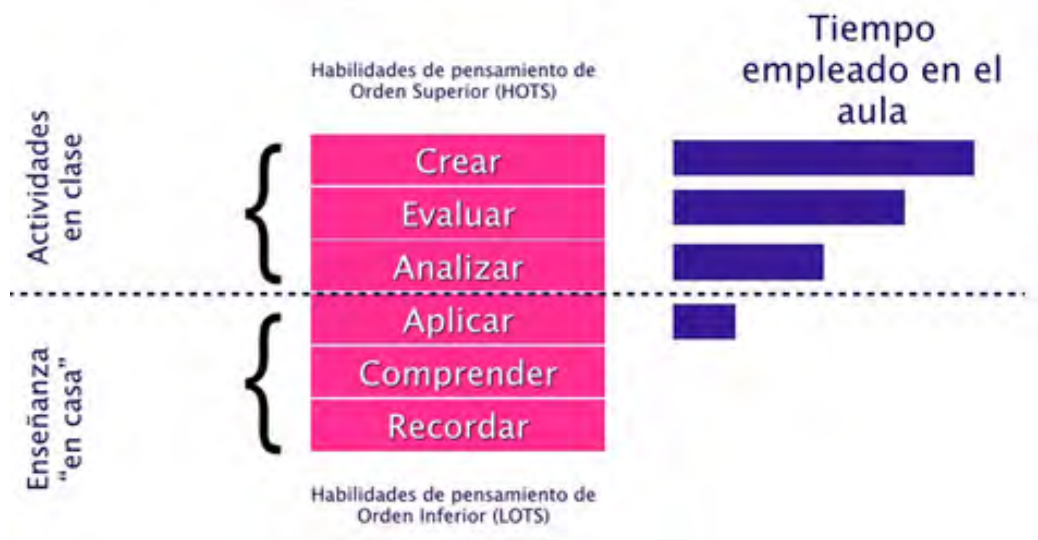

Figura 1. Tiempo utilizado en la "clase invertida" por taxonomía de Bloom. Tourón, J., Santiago, R., y Diez, A. (2014)

Rosenberg (2013) relata la experiencia llevada a cabo en Clintondale High School, primer centro educativo de los Estados Unidos en implantar el modelo "flipped classroom" en todas sus aulas, mostrándonos que con esta implantación, el tiempo de atención individualizada del docente al alumnado se ha multiplicado por cuatro, y se han elevado los niveles de rendimiento y de motivación de dicho alumnado.

Son muchas las investigaciones que recogen evidencias empíricas sobre el potencial del modelo "flipped classroom" para mejorar tanto la motivación, como el rendimiento de los estudiantes en educación superior y en los estudios de educación (Bergmann y Sams, 2012; Rosemberg, 2013; Tourón y Santiago, 2015; Miragall y García-Soriano, 2016; Palomares y Cebrián, 2016; Aguilera et al., 2017; Contreras et al., 2017; Matzumura et al., 2018; Awidi y Paynter, 2018; Hinojo et al., 2018). Resumiendo, las características asociadas a este método se corresponden con el aumento de las horas dedicadas al aprendizaje, el uso de la tecnología, protagonismo del alumnado, la inversión de roles y el compromiso por parte del docente y discente para llevar a cabo el proceso de aprendizaje, favoreciendo la autorregulación del aprendizaje y generando un ambiente colaborativo en el aula. Además, como especifican Cebrián et al. (2019) este modelo puede resultar idóneo para el desarrollo de talento de los más capaces.

Razonadamente, el modelo conlleva también algunas desventajas con las que debemos contar a la hora de su aplicación para poder eliminarlas del mejor modo posible. Algunos autores (Aguilera et al., 2017) destacan la reticencia del alumnado, que puede optar por preferir el método tradicional, negándose así a abandonar su zona de confort. Asimismo, Jordán-Lluch et al. (2014), subrayan el esfuerzo que debe hacer el docente, ya que debe crear nuevos materiales y modificar las programaciones de las asignaturas que imparte, y el bajo grado de competencia digital que puede poseer el alumnado y falta de habilidades comunicativas en el profesorado. 
Por ello, destacamos el interés de este estudio y de la temática para poder ratificar y comprobar de manera empírica estos resultados, que a priori remarcan que las calificaciones mejoran en el alumnado con respecto al modelo tradicional de enseñanza, al igual que la motivación gracias a los materiales y recursos audiovisuales con los que se trabajan.

\section{Objetivos}

El objetivo general de este estudio ha sido realizar un pilotaje en la asignatura Tendencias Contemporáneas de la Educación del Grado de Maestro de Educación Primaria de la Universidad de Castilla-La Mancha y evaluar el grado de satisfacción del alumnado sobre la metodología empleada. Como objetivos específicos se destacan: a) Identificar con qué metodología el alumnado considera que asimila mejor los contenidos, si con la clase invertida o con la clase tradicional; b) Analizar las ventajas y los inconvenientes que los estudiantes perciben con la implementación de esta metodología; c) Señalar cuáles son las actividades o recursos audiovisuales que resultan más útiles para mejorar el proceso de aprendizaje; d) Considerar si con este modelo aumenta el rendimiento y las calificaciones del alumnado en la enseñanza superior.

\section{Método}

Diseño

A fin de cumplir los objetivos del presente trabajo se planteó un estudio cuantitativo, no experimental, descriptivo, donde la población fue el alumnado de Grado de Maestro.

\section{Participantes}

La muestra de estudio han sido estudiantes matriculados en la asignatura de Tendencias Contemporáneas de la Educación de segundo curso del Grado de Maestro en Educación Primaria de la Facultad de Educación. Se recogió una muestra de 82 alumnos y alumnas de la Universidad de Castilla-La Mancha y la experiencia docente se desarrolló durante el segundo cuatrimestre del curso académico 2018-2019. De un total de 97 estudiantes matriculados en la asignatura, participaron voluntariamente en el estudio 82 (85.5\%). De esta muestra de estudiantes, 39 fueron hombres $(47,56 \%)$ y 43 mujeres $(52,44 \%)$.

\section{Instrumento de recolección de datos}

La recogida de datos se llevó a cabo tras 12 semanas de la implementación de esta metodología. Se distribuyó de forma online un cuestionario. El cuestionario empleado en este estudio para evaluar sus percepciones sobre el entorno de aprendizaje invertido en el aula fue diseñado por Gilboy et al. (2015). El cuestionario consiste en una escala Likert, instrumento con respuestas anónimas y cada una con cinco ítems con las siguientes opciones: muy de acuerdo, de acuerdo, neutral, en desacuerdo o 
muy en desacuerdo. Este cuestionario presenta un Alfa de Cronbach con un valor de 0.71, un valor aceptable para la confiabilidad según George y Mallery (2002). El cuestionario también incluyó dos preguntas abiertas sobre las opiniones de los estudiantes sobre el trabajo antes y durante la clase invertida.

\section{Procedimiento}

Puesta en marcha del aula invertida

El primer día de clase se planteó a los estudiantes la posibilidad de utilizar esta nueva metodología frente a la clase tradicional. Se les explicó cómo sería la dinámica de las clases, informando que con el modelo "flipped classroom" se invierten los roles en el aula; es decir, el estudiante adquiere un rol principal en su proceso de aprendizaje y el docente actúa como guía (Bergmann y Sams, 2012), y que sería necesario dedicar un tiempo en casa para revisar el material. La totalidad de la clase estuvo de acuerdo en emplear esta metodología y se comprometieron a trabajar en casa.

El segundo paso, fue facilitar al alumnado en la primera semana de clase, todo el material necesario para trabajar el temario en casa, que sería consultado fuera del horario lectivo por parte del alumnado, destinando las horas de docencia en el aula a la resolución de dudas (Castilla et al., 2015). Los contenidos docentes de la asignatura estuvieron expuestos en la plataforma virtual de la Universidad de Castilla-La Mancha, para que el alumnado pudiera tener acceso a ellos desde cualquier punto con acceso a internet.

El docente preparó vídeos explicativos del temario y aportó material adicional para su estudio, mientras que el alumnado se involucró totalmente en su proceso de aprendizaje (Gonçalves y Quaresma, 2018). Se fomentó, en todo momento, la comunicación con los alumnos y alumnas, tanto sincrónica como asincrónica, para mantener una relación alumnado-profesorado más interactiva, considerando siempre las actividades presenciales. Lo que destaca de este modelo es que permite al estudiante acceder al contenido de forma ubicua, es decir, en cualquier momento y lugar gracias al uso de la tecnología, prescindiendo de la figura del profesor como transmisor de contenidos (Vidal et al., 2016).

Además, se llevó a cabo un seguimiento continuo y feed-forward de las actividades realizadas, para detectar las fortalezas y debilidades del proceso de aprendizaje y poder establecer medidas correctoras o estrategias de mejora sobre la marcha. Dichas actividades se fundamentaron en el trabajo en equipo y colaborativo, fomentando las búsquedas de información autónomas. Entre dichas actividades caben destacar: lecturas de documentos, artículos, visionado de vídeos, manejo de páginas web, búsquedas autónomas de información, tertulias dialógicas pedagógicas, grupos interactivos, etc. En el aula se Ilevaron a cabo presentaciones orales y representaciones de roles.

Asimismo, los trabajos de los estudiantes fuera del aula y los elementos multimedia que trabajaron en las asignaturas objeto de estudio han sido: Visionado de vídeos como cortometrajes, monografías, reportajes, etc., especialmente de YouTube. El uso 
del vídeo proporciona una serie de ventajas tales como la posibilidad de visualizar los contenidos tantas veces como se desee, permite actualizar el contenido constantemente, posibilita su uso en los años siguientes y, probablemente, hablemos de uno de los recursos más dinámicos que existen (Lara y Rivas, 2009). También el manejo de páginas webs y blogs educativos, casos prácticos a resolver tales como el de búsquedas de información sobre determinadas metodologías activas innovadoras en el aula como aprendizaje cooperativo, comunidades de aprendizaje, etc. Lecturas de documentos (expuestos en la plataforma virtual de la asignatura): Pedagogía del Oprimido de Paulo Freire, por ejemplo. Realización de una tertulia dialógica pedagógica sobre temas actuales de educación que aparecen en el temario, así como la organización de grupos interactivos sobre otros aspectos que el alumnado ha trabajado en casa o en horario no lectivo. Grupos de discusión. Todas estas actividades estuvieron relacionadas con los niveles de la taxonomía de aprendizaje de Bloom (Figura 2).

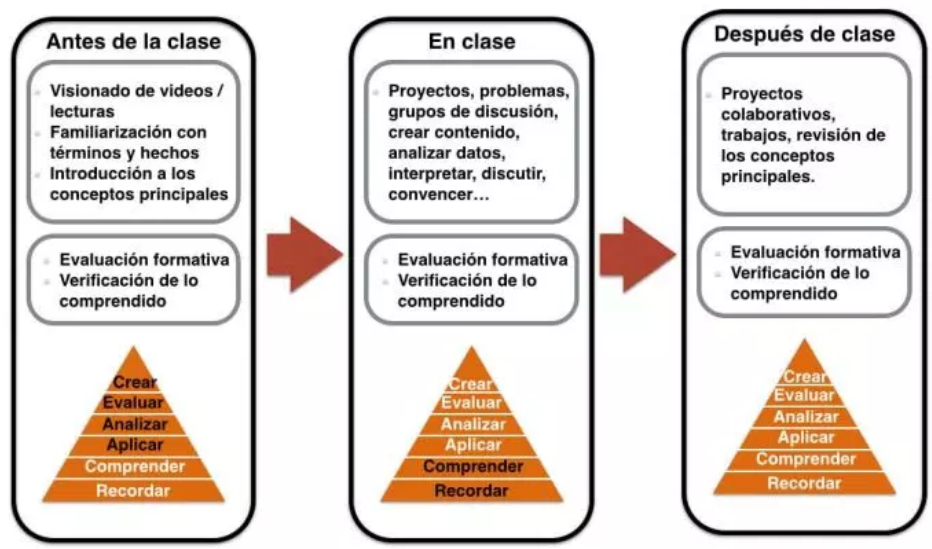

Figura 2. Actividades para "flipped classroom" antes, durante y después de la clase y su relación con los niveles de la taxonomía de aprendizaje revisada de Bloom (en Boslaugh, S. P. (2013)). Fuente: theflippedclassroom.es

El primer día de la semana que había clase de la asignatura se empezaba realizando una revisión del tema o unidad didáctica que se había trabajado y analizado por el alumnado, siguiendo una serie de preguntas elaboradas por el docente $y$, a partir de dichas respuestas, se comenzaba un debate de los contenidos mediante preguntas y respuestas. El docente exponía brevemente un resumen del tema y trataba de resolver todas las dudas que surgían en el aula tras haber trabajado el tema en sus casas. El segundo día de la semana de clase se dedicaba a realizar trabajo activo en grupos de cuatro a cinco integrantes, que contemplaban resolución de casos prácticos y discusión en grupo. Los estudiantes accedían a información mediante smartphone, PC y/o dossier de la asignatura. Una vez finalizada la clase se sugería a los estudiantes que profundizaran sobre los temas tratados durante la clase y los complementaran con información adicional (videos alojados en YouTube). En la resolución de los casos prácticos elaborados por los estudiantes se realizaba la retroalimentación y calificación formativa de los mismos (Tabla 2). 
Terminada la experiencia, tras 12 semanas se envía al alumnado el enlace para poder rellenar el cuestionario online y su posterior análisis de los resultados.

Tabla 2. Desarrollo de la propuesta de innovación "flipped classroom" o "aula invertida"

\begin{tabular}{|c|c|c|c|}
\hline \multicolumn{2}{|c|}{ Antes de la clase } & \multicolumn{2}{|c|}{ Durante la clase "flipped classroom" } \\
\hline Docente & Estudiante & Docente & Estudiante \\
\hline $\begin{array}{l}\text { Preparación de mate- } \\
\text { rial } \\
\text { Documentos y en- } \\
\text { laces colgados en la } \\
\text { Plataforma Virtual de } \\
\text { la asignatura }\end{array}$ & $\begin{array}{l}\text { Acercamiento a los } \\
\text { conceptos principales } \\
\text { del tema } \\
\text { Lectura de los textos } \\
\text { propuestos y visio- } \\
\text { nado de videos de la } \\
\text { Plataforma Virtual de } \\
\text { la asignatura }\end{array}$ & $\begin{array}{l}\text { Repaso de los con- } \\
\text { ceptos del tema expli- } \\
\text { cados anteriormente } \\
\text { Exposición de las acti- } \\
\text { vidades y casos prác- } \\
\text { ticos a realizar } \\
\text { Revisión y corrección } \\
\text { de dichas actividades } \\
\text { y casos prácticos. Re- } \\
\text { solución de dudas }\end{array}$ & $\begin{array}{l}\text { Planteamiento de du- } \\
\text { das surgidas en casa } \\
\text { del estudio de los } \\
\text { conceptos del tema } \\
\text { Realización de las } \\
\text { actividades y casos } \\
\text { prácticos de manera } \\
\text { individual y/o grupal } \\
\text { según corresponda } \\
\text { Discusión }\end{array}$ \\
\hline
\end{tabular}

\section{Resultados}

Se ha realizado un análisis descriptivo de las frecuencias y porcentajes de las puntuaciones obtenidas por el alumnado tras la administración del cuestionario, mostrando los resultados obtenidos en la Tabla 3.

Los valores obtenidos en la Tabla 3, demuestran que a un $81,6 \%$ de los estudiantes les gustó la posibilidad de ver un vídeo en vez de tener una clase tradicional (conferencia) de los temas de la asignatura. Al igual que la mayoría, un 85,3\%, también prefiere realizar trabajos activos y grupales en clases como los que se Ilevaron a cabo con metodología "flipped classroom" o "aula invertida" a tener la clase tradicional (conferencia) del profesor. En relación con el material empleado, un 82,9\% del alumnado considera que el uso de vídeos le permite aprender el material de estudio más eficazmente que hacer las lecturas en solitario. En cuanto a la asimilación de contenidos, un 75,6\% considera que aprendió más cuando utilizó el método de aprendizaje "flipped classroom" o "aula invertida" (vídeos, lecturas cortas y actividades de aprendizaje activo en clase) en comparación con el método tradicional (exposición del profesor). Por último, un 14,6\% de los alumnos y alumnas sugieren que se sentían desconectados sin un profesor que les guiase cuando consultaban el material en casa.

Por otra parte, si nos detenemos en los comentarios abiertos del cuestionario podemos comprobar que al alumnado lo que más le gustó fue el poder trabajar a su ritmo y adaptándose al tiempo y a sus necesidades (35,5\%), especialmente para aqueIlos alumnos y alumnas que, por diversos motivos, no pueden asistir regularmente a clase. Igualmente, se revelan preocupaciones en el alumnado relativas a la ausencia del profesorado para poder resolver las dudas y preguntas que le surgían fuera del aula (22,1\%). Asimismo, uno de los aspectos que también destacan en estas observaciones es que a la hora de realizar las actividades grupales que se realizan en clase, muchos de los compañeros y compañeras no sabían trabajar activamente con este 
modelo $(15,6 \%)$ y, un $47,2 \%$, reconoce que pocas veces dedica el tiempo necesario a los visionados y lecturas a realizar en la casa previas a las clases.

Tabla 3. Análisis descriptivo del cuestionario sobre "aula invertida"

\begin{tabular}{|c|c|c|c|c|c|c|c|c|c|c|c|}
\hline \multirow[t]{2}{*}{ Ítems } & \multirow[t]{2}{*}{$\mathbf{n}$} & \multicolumn{2}{|c|}{$\begin{array}{c}\text { Muy en } \\
\text { desacuerdo }\end{array}$} & \multicolumn{2}{|c|}{$\begin{array}{c}\text { En } \\
\text { desacuerdo }\end{array}$} & \multicolumn{2}{|c|}{ Neutral } & \multicolumn{2}{|c|}{$\begin{array}{c}\text { De } \\
\text { acuerdo }\end{array}$} & \multicolumn{2}{|c|}{$\begin{array}{l}\text { Muy de } \\
\text { acuerdo }\end{array}$} \\
\hline & & $\Sigma$ & $\%$ & $\sum$ & $\%$ & $\sum$ & & $\sum$ & $\%$ & $\sum$ & $\%$ \\
\hline $\begin{array}{l}\text { Ítem } 1 . \text { Me gustó la posibili- } \\
\text { dad de ver un vídeo en vez } \\
\text { de tener una clase tradicional } \\
\text { (conferencia) de los temas } \\
\text { del curso }\end{array}$ & 82 & & & 2 & 2,4 & 1315 & 5,8 & 22 & 26,8 & 45 & 54,8 \\
\hline $\begin{array}{l}\text { Ítem 2. Prefiero tener la clase } \\
\text { tradicional (conferencia) del } \\
\text { profesor en vez de realizar tra- } \\
\text { bajos activos y grupales en cla- } \\
\text { ses como los que se llevaron a } \\
\text { cabo con metodología FC }\end{array}$ & 82 & 53 & 64,6 & 17 & 20,7 & $10 \quad 12$ & 2,1 & 2 & 2,4 & & \\
\hline $\begin{array}{l}\text { Ítem } 3 \text {. El uso de vídeos me } \\
\text { permite aprender el material } \\
\text { de estudio más eficazmente } \\
\text { que hacer las lecturas en so- } \\
\text { litario }\end{array}$ & 82 & & & 2 & 2,4 & 121 & 4,6 & 27 & 32,9 & 41 & 50 \\
\hline $\begin{array}{l}\text { Ítem 4. Yo aprendí más } \\
\text { cuando utilicé el método de } \\
\text { aprendizaje FC (vídeos, lec- } \\
\text { turas cortas y actividades de } \\
\text { aprendizaje activo en clase) } \\
\text { en comparación con el mé- } \\
\text { todo tradicional (exposición } \\
\text { del profesor). }\end{array}$ & 82 & 1 & 1,2 & 4 & 4,8 & $\begin{array}{l}1 \\
18,2\end{array}$ & 5 & 31 & 37,8 & 31 & 37,8 \\
\hline $\begin{array}{l}\text { Ítem } 5 \text {. Me sentía desconec- } \\
\text { tado sin un profesor presente } \\
\text { durante los vídeos o activida- } \\
\text { des virtuales. }\end{array}$ & 82 & 24 & 29,2 & 35 & 42,6 & $\begin{array}{l}1 \\
13,4\end{array}$ & 1 & 6 & 7,3 & 6 & 7,3 \\
\hline
\end{tabular}

Con respecto a las calificaciones obtenidas y comparadas con años anteriores, cursos 2017/18 y 2018/19, no se han obtenido diferencias estadísticamente significativas, lo que no significa que este modelo no interese para el aprendizaje, ya que podría deberse en particular a los estudiantes que son diferentes en cada uno de los cursos y que podrían ser de un corte especialmente tradicional o con poco desarrollo de la competencia digital y tratamiento de la información. Otros aspectos que podrían influir también son las aptitudes cognitivas, motivación, relaciones dentro del grupo clase, etc. 
Si lo comparamos con respecto al primer cuatrimestre que no se aplicó la metodología con el segundo cuatrimestre en la que se implementó este modelo, los resultados tampoco han sido bastante significativos, ya que prácticamente no hubo diferencias en la adquisición de los aprendizajes, aunque aumentaron levemente las calificaciones en el segundo cuatrimestre del curso 2018/19.

\section{DisCUSIÓN Y CONCLUSIONES}

EI EEES se fundamenta en un enfoque basado en las competencias, definidas como el conjunto de conocimientos, destrezas y actitudes que deben movilizarse en contextos de ejecución determinados, y que deben ser adquiridas por el alumnado. Uno de los aspectos que se destaca es el uso de metodologías que contribuyan al desarroIlo de dichas competencias y que deben ser activas, participativas, colaborativas, que planteen problemas de la vida real y que se pueda trabajar a través de proyectos. Sin embargo, en la realidad de las aulas universitarias se observa que predominan las clases magistrales y expositivas, simplemente unido al desarrollo de una serie de "prácticas", en muchas ocasiones desvinculadas de la parte teórica, que se convierten en una evaluación acumulativa de tareas que se aleja del carácter formativo que el EEES defiende, y que no llevan aparejadas la necesaria retroalimentación indispensable para una adquisición real y progresiva de la competencia deseada (Pérez Juste, 2013).

El modelo "flipped classroom" o "aula invertida" puede ser la alternativa a para poder cumplir todos los principios metodológicos que defiende el EEES, y que verdaderamente sirva para desarrollar las competencias en el alumnado. En efecto, se han dado muchas investigaciones que refuerzan nuestro estudio, que trataba de ver el grado de satisfacción del alumnado universitario ante el modelo "flipped classroom" o "aula invertida", ofreciendo resultados similares a los obtenidos por Faundez et al., (2016); Palomares y Cebrián (2016) y García y Cremades (2019). Razonadamente, el desarrollo de esta experiencia ha resultado ser muy positiva, tanto en el proceso de aprendizaje como en la aceptación por parte del alumnado, siendo claves el uso de recursos audiovisuales para el proceso de aprendizaje, lo que evidencia la necesidad de la inclusión de este tipo de metodologías, así como otras Tecnologías de la Información y de la Comunicación, para el proceso de enseñanza-aprendizaje en las aulas universitarias. Por parte del alumnado también hemos visto una elevada aceptación y satisfacción, lo que señala que se puede llevar a cabo y generalizar en otras asignaturas de la carrera dada su eficacia y utilidad frente a la clase tradicional y magistral.

Se ha evidenciado que el papel del docente es importante, y ello ha quedado demostrado en las respuestas que da el alumnado cuando se les pregunta por la asimilación de contenidos, y cómo se enfrenta en casa a la materia.

En cuanto a los materiales que se ofrecen con este modelo, destacan el visionado de videos, lo que nos Ilevaría a pensar en implicaciones educativas dentro del ámbito universitario. También es cierto que el tiempo que necesita el profesorado en realizar y/o preparar todo el material así como organizarlo y colgarlo en la Plataforma Virtual, es costoso y a esto se une la escasez de recursos humanos y materiales que tiene la 
universidad en estos últimos años y la excesiva carga docente en algunas universidades españolas.

Otro de los aspectos que se destacan en el estudio es que un alto porcentaje de alumnado reconoce no dedicar el tiempo necesario en casa al estudio de los documentos y visionado de los vídeos que el profesorado propone para el trabajo en casa. Desde el primer día y en consonancia con otros estudios relacionados con el tema (Palomares y Cebrián, 2016) es recomendable que el alumnado conozca a lo que se compromete con esta metodología y, aun haciéndolo como se ha descrito anteriormente, los resultados no han sido del todo positivos en este aspecto. Esto puede ser debido al material utilizado, ya que para muchos autores como O’Flaherty y Philips (2015), inciden en que si el material que se utiliza carece de interactividad el alumnado no participará en las tareas que se manden para realizar en casa, algo que hace pensar y replantear el material utilizado, prevaleciendo el tratamiento de documentos escritos que podían haber sido reemplazados por conferencias en diferido sobre el tema a tratar en el aula. No se debe olvidar, como también especifican Tiahrt y Jason (2016), que ha que centrarse, no sólo en el material que se prepara para el trabajo individual en casa, sino que no se debe descuidar la organización y desarrollo de las actividades y tareas que hagamos en el aula universitaria.

Resulta preciso reconocer que esta investigación no carece de limitaciones. En el estudio solamente nos hemos centrado en la satisfacción del alumnado con respecto a su experiencia con este modelo, pudiendo haber evaluado también la satisfacción del profesorado implicado y haber profundizado -aún más- en los resultados académicos y en la correlación de las preguntas mostradas en los exámenes con la metodología utilizada en el aula.

Igualmente, el estudio se podía haber enriquecido más con otro grupo de control en el que se desarrollase la metodología tradicional y expositiva, pero proporciona un punto de partida para futuras investigaciones.

Los resultados obtenidos nos animan a seguir trabajando en este sentido y a corroborar que la metodología del "flipped classroom" o "aula invertida" es útil para el alumnado, respeta su ritmo de aprendizaje y despierta su motivación por el estudio. Entre algunos de los aspectos novedosos que aporta esta investigación destacamos que conseguimos un aula universitaria activa, participativa e inclusiva, adaptada a todo el alumnado y que convierte una asignatura de corte teórico en otra más práctica y motivadora en el ámbito específico de los estudios del Grado de Maestro.

\section{REFERENCIAS BIBLIOGRÁFICAS}

Aguilera, C., Manzano, A., Martínez, I., Lozano, M. C. y Casiano, C. (2017). El modelo flipped classroom. International Journal of Developmental and Educational Psychology, 4(1), 261-266. https://doi.org/10.17060/ijodaep.2017.n1.v4.1055

Ash, K. (2012). Educators evaluate "flipped classrooms". Education Week, 32(2), s6-s8. 
Awidi, I. T. y Paynter, M. (2018). The impact of a flipped classroom approach on student learning experience. Computers \& Education, 128, 269-283. https://doi. org/10.1016/j.compedu.2018.09.013

Aznar, I., Cáceres, M. P. y Romero, J. M. (2018). Indicadores de calidad para evaluar buenas prácticas docentes de mobile learning en educación superior. Education in the Knowledge Society (EKS), 19(3), 53-68. https://doi.org/10.14201/ eks20181935368

Bergmann, J. y Sams, A. (2012). Flip Your Classroom: Reach Every Student in Every Class Every Day (pp. 120-190). International Society for Technology in Education.

Bergmann, J. y Sams, A. (2014). Dale la vuelta a tu clase. Lleva tu clase a cada estudiante, en cualquier momento y cualquier lugar. SM ( $\left.2^{\mathrm{a}} \mathrm{ed}\right)$.

Castilla, G., Alriols, J., Romana, M. y Escribano, J. J. (2015). Resultados del estudio experimental de flipped learning en el ámbito de la enseñanza de matemáticas en ingeniería. En M. A. Ruiz Rosillo (Coord.), Actas de las XII Jornadas Internacionales de Innovación Universitaria (pp. 774-782). Universidad Europea de Madrid.

Cebrián-Martínez, A., Palomares-Ruiz, A. y García-Perales, R. (2019). El aprendizaje autorregulado y su efecto en el rendimiento académico. Descripción de una experiencia con los alumnos del Grado de Maestro de la Facultad de Educación de Albacete (estudio intersujetos). En R. Roig-Vila (Ed.), Investigación e Innovación en la Enseñanza Superior. Nuevos contextos, nuevas ideas (pp. 66-79). Ediciones Octaedro.

Contreras, J. A., Arias, J., Melo, M. G. y Martín, R. (2017). Uso del modelo de aprendizaje inverso para mejorar materiales educativos universitarios. Revista lbérica de Sistemas y Tecnologías de Información, 23, 17-32. https://doi.org/10.17013/ risti.23.17-32

Faundez, A. R. O., Bastias, J. M. A. y Polanco, M. P. R. (2016). Evaluación de metodología flipped classroom: Primera experiencia. Innoeduca. International Journal of Technology and Educational Innovation, 2(2), 90-99.

García-Gil, D. y Cremades-Andreu, R. (2019). Flipped classroom en educación superior: Un estudio a través de relatos de alumnos. Revista Mexicana de Investigación Educativa, 24(80), 101-123.

George, D. y Mallery, P. (2003). SPSS for Windows step by step: A simple guide and reference. 11.0 update. Allyn \& Bacon.

Gilboy, M. B., Heinerichs, S. y Pazzaglia, G. (2015). Enhancing student engagement using the flipped classroom. Journal of Nutrition Education and Behavior, 47(1), 109-114. https://doi.org/10.1016/j.jneb.2014.08.008

Gonçalves, Z. T. y Quaresma, D. (2018). Metodologia activa: Sala de aula invertida e suas práticas na educação básica. REICE. Revista Iberoamericana sobre Calidad, Eficacia y Cambio en Educación, 16(4), 63-78. https://doi.org/10.15366/reice2018.16.4.004 
Hernández, C. y Tecpan, S. (2017). Aprendizaje por indagación para la construcción de arquetipos en física; el caso de un curso para formación de profesores en Chile. Latin-American Journal of Physics Education, 11(2).

Hinojo, F. J., Mingorance, A. C., Trujillo, J. M., Aznar, I. y Cáceres, M. P. (2018). Incidence of the flipped classroom in the physical education students academic performance in university contexts. Sustainability, 10(5), 1-13. https://doi. org/10.3390/su10051334

Jordan-Lluch, C., Pérez Peñalver, M. y Sanabria-Codesal, E. (2014). Flipped classroom: Reflexiones y opiniones de los implicados. En Jornadas de Innovación Educativa y docencia en Red de la Universitat Politècnica de València (pp. 310-323). Universitat Politècnica de València. https://doi.org/10.1109/isie.2007.4375002

Lara, S. y Rivas, S. (2009). Aprendizaje autorregulado y fomento de competencias en dos asignaturas de máster a través del empleo de plantillas de evaluación, método del caso, role-playing y video digital. Revista Educación $X X I, 12,67-96$.

Matzumura, J. P., Gutiérrez, H., Zamudio, L. A. y Zavala, J. C. (2018). Aprendizaje invertido para la mejora y logro de metas de aprendizaje en el curso de metodología de la investigación en estudiantes de universidad. Revista Electrónica Educare, 22(3), 1-21. https://doi.org/10.15359/ree.22-3.9

Miragall, M. y García-Soriano, G. (2016). Transformando una clase del grado en psicología en una flipped classroom. @TIC. Revista d'Innovació Educativa, 17, 21 29. https://doi.org/10.7203/attic.17.9097

O'Flaherty, J. y Phillips, C. (2015). The use of flipped classrooms in higher education: A scoping review. The Internet and Higher Education, 25, 85-95.

Palomares Ruiz, A. y Cebrián Martínez, A. (2016). Una experiencia de Flipped Classroom o Aula Invertida en la Facultad de Educación de Albacete. En R. Roig-Vila (Coord.), Tecnología, innovación e investigación en los procesos de enseñanza-aprendizaje, (pp. 2860-2871). Ediciones Octaedro.

Pérez Juste, R. (2013). Evaluación Institucional al Servicio de la Calidad de la Organización Educativa. I Jornadas Internacionales sobre liderazgo y calidad de la educación. Mayo. (pp. 17-24).

Reyes, A. E. (2015). Educación y formación en la Unión Europea: Análisis del proceso de Bolonia, el espacio europeo de educación superior, la estrategia Europa 2020 y el programa Erasmus+. Derecho y Cambio Social, 12(42), 1-23.

Rosenberg, T. (2013). Turning Education Upside Down. http://opinionator.blogs. nytimes.com/2013/10/09/turning-education-upside-down/

Santiago, R. (2013). The Flipped classroom. https://www.theflippedclassroom.es/

Santiago, R. y Bergmann, J. (2018). Aprender al revés. Flipped Learning 3.0 y metodologías activas en el aula. Paidós Educación. 
Tiahrt, T. y Jason, C. P. (2016). What Do I Do with this Flipping Classroom: Ideas for Effectively Using Class Time in a Flipped Course. Business Education Innovation Journal 8(2), 85-91.

Tourón, J. (2013). The Flipped Classroom: ¿no has 'flipado' aún? http://www.javiertouron.es/2013/06/the-flipped-classroom-no-has-flipado.html

Tourón, J. y Santiago, R. (2015). El modelo flipped learning y el desarrollo del talento en la escuela. Revista de Educación, 368, 196-231. https://doi.org/10.4438/1988592X-RE-2015-368-288.

Tourón, J., Santiago, R. y Diez A. (2014). The Flipped Classroom: Cómo convertir la escuela en un espacio de aprendizaje. Grupo Océano.

Tucker, B. (2012). The flipped classroom. Education Next, 12(1), 82-83. http://educationnext. org/the-flipped-classroom

Vidal, M., Rivera, N., Nolla, N., Morales, I. R. y Vialart, M. N. (2016). Aula invertida, nueva estrategia didáctica. Educación Médica Superior, 30(3), 1-12. 